\title{
Few femtosecond, few kiloampere electron bunch produced by a laser-plasma accelerator
}

\author{
O. Lundh ${ }^{1 \star \dagger}$, J. Lim ${ }^{1}$, C. Rechatin ${ }^{1}$, L. Ammoura ${ }^{1}$, A. Ben-Ismaïl ${ }^{2}$, X. Davoine ${ }^{3}$, G. Gallot ${ }^{4}$, J-P. Goddet ${ }^{1}$, \\ E. Lefebvre ${ }^{3}$, V. Malka ${ }^{1 \star}$ and J. Faure ${ }^{1}$
}

Particle accelerators driven by the interaction of ultraintense and ultrashort laser pulses with a plasma' $^{1}$ can generate accelerating electric fields of several hundred gigavolts per metre and deliver high-quality electron beams with low energy spread $^{2-5}$, low emittance ${ }^{6}$ and up to $1 \mathrm{GeV}$ peak energy 7,8 . Moreover, it is expected they may soon be able to produce bursts of electrons shorter than those produced by conventional particle accelerators, down to femtosecond durations and less. Here we present wide-band spectral measurements of coherent transition radiation which we use for temporal characterization. Our analysis shows that the electron beam, produced using controlled optical injection ${ }^{9}$, contains a temporal feature that can be identified as a $15 \mathrm{pC}, 1.4-1.8 \mathrm{fs}$ electron bunch (root mean square) leading to a peak current of 3-4 kA depending on the bunch shape. We anticipate that these results will have a strong impact on emerging applications such as short-pulse and short-wavelength radiation sources ${ }^{10,11}$, and will benefit the realization of laboratory-scale free-electron lasers ${ }^{12-14}$.

The ponderomotive force generated by the focused laser pulse is proportional to the gradient of the laser intensity. It pushes electrons out of regions of high intensity and separates them from the ions, thus creating a plasma wave that propagates in the wake of the laser pulse with a phase velocity close to $c$, the speed of light in vacuum. The characteristic length of the accelerating cavity that forms behind the driving laser pulse is the plasma wavelength $\lambda_{\mathrm{p}}$. In a typical laser wakefield acceleration experiment, $\lambda_{\mathrm{p}}=10-30 \mu \mathrm{m}$ for plasma densities $n_{\mathrm{e}}=10^{18}-10^{19} \mathrm{~cm}^{-3}$. The electric field changes along the length of the plasma wave, therefore, to generate an electron beam with low energy spread and low divergence, the electron bunch should reside within the focusing and accelerating phase of the wave, which has a length $\lambda_{\mathrm{p}} / 4$. These heuristic arguments indicate that one would expect the bunch duration to be ultrashort, $\tau<\lambda_{\mathrm{p}} / 4 c \approx 10$ fs (refs 9,15). However, to the best of our knowledge, such short durations have not previously been directly measured.

Traditional techniques to measure the electron bunch duration, such as streak cameras and radio-frequency sweeping cavities, do not have the temporal resolution required for femtosecond bunches. Therefore, we have employed a method in the frequency domain and measured the coherent transition radiation (CTR) that is emitted by the electron bunch as it passes through a thin metallic foil.

CTR is an established particle beam diagnostic and has been used to diagnose micro-structures of picosecond-bunches ${ }^{16,17}$ and for benchmarking simulations of femtosecond-bunch dynamics

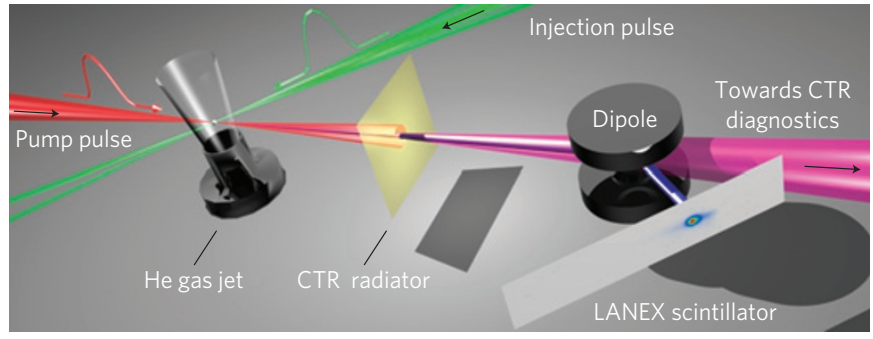

Figure 1 | Experimental arrangement. An ultrashort and ultraintense laser pulse (red) is focused on a gas jet in which a plasma wave is excited. Electrons are injected into the plasma wave during the collision with the injection pulse (green), which arrives at a relative angle of $135^{\circ}$. The accelerated electron beam (blue) traverses an aluminium foil and generates transition radiation in a narrow cone along the forward direction (violet). The electron beam is analysed by means of a magnetic dipole and a phosphor screen ${ }^{28}$ whereas the transition radiation exits the interaction chamber for characterization.

in a multi-gigaelectronvolt radio-frequency accelerator ${ }^{18}$. CTR at terahertz frequencies was used for temporal characterization of laser-accelerated electron bunches with duration longer than 30 fs (refs 19-21). Here we employ near- to mid-infrared CTR spectroscopy for measurement of laser-accelerated bunches with durations of only a few femtoseconds.

For a monoenergetic electron beam, the angular radiation field is a hollow cone with half opening angle $\theta=1 / \gamma$, where $\gamma=1 / \sqrt{1-(v / c)^{2}}$ is the relativistic factor and $v$ is the electron velocity. The spectral radiation field at frequency $\omega$ and observation angle $\theta$, is given by ${ }^{22}$

$$
\frac{\mathrm{d}^{2} W}{\mathrm{~d} \omega \mathrm{d} \Omega}=\left[N+N^{2} F(\omega, \theta)\right] \frac{\mathrm{d}^{2} w}{\mathrm{~d} \omega \mathrm{d} \Omega}
$$

where $\mathrm{d}^{2} w / \mathrm{d} \omega \mathrm{d} \Omega$ is the transition radiation spectrum emitted by a single electron, $\Omega$ is the solid angle and $N$ is the number of electrons in the bunch. The form factor $F(\omega, \theta)=$ $\left|\int f(\mathbf{x}) \exp (-i \mathbf{k} \cdot \mathbf{x}) \mathrm{d}^{3} \mathbf{x}\right|^{2}$ is the square amplitude of the Fourier transform of the normalized electron bunch distribution, $f(\mathbf{x})$, and $\mathbf{k}$ is the radiation wavenumber in the direction of observation. The radiation is said to be coherent if the second term in equation (1) dominates. This typically occurs only if the electron bunch length is comparable to or less than the radiation wavelength observed. In this case, electrons radiate in phase and the radiation is amplified coherently.

\footnotetext{
${ }^{1}$ Laboratoire d'Optique Appliquée, ENSTA, Ecole Polytechnique, CNRS, 91761 Palaiseau, France, ${ }^{2}$ Laboratoire Leprince Ringuet, Ecole Polytechnique, UMR 7638, 91128 Palaiseau, France, ${ }^{3}$ CEA, DAM, DIF, Bruyères-le-Châtel, 91297 Arpajon, France, ${ }^{4}$ Laboratoire d'Optique et Biosciences, CNRS, INSERM, Ecole Polytechnique, ENSTA, Palaiseau, France. †Present address: Lund University, Sweden. *e-mail: olle.lundh@fysik.lth.se; victor.malka@ensta.fr.
} 


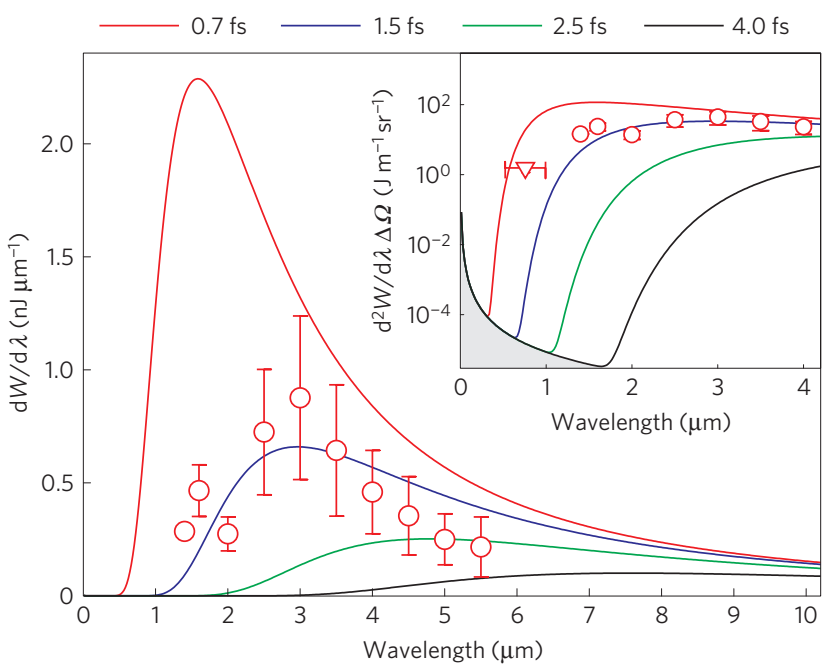

Figure 2 | Measured CTR spectrum. Experimental spectrum using the infrared monochromator (circles) and the visible spectrometer (triangles), and analytical CTR spectra for different bunch durations (solid lines). Error bars indicate shot fluctuations and spectral range of the measurement. The bunch shape is taken to be Gaussian with r.m.s. duration, $\sigma$, as indicated by the legend. Other bunch characteristics used for the calculation are the experimentally measured mean values. Data in the inset has been divided by the solid angle of respective instrument. Grey area indicates the incoherent intensity level.

Equation (1) also shows that the shape of the CTR spectrum contains information on the shape of the electron bunch. By independently measuring the bunch charge, energy spectrum and transverse distribution, it is possible to fit the theoretical model to the experimental results and retrieve information on the bunch shape ${ }^{23}$.

To generate high-quality and stable electron bunches, we use the colliding pulse injection scheme ${ }^{9}$. The $30 \mathrm{fs}$ (full-width at half-maximum (FWHM)) pump pulse and injection pulse collide at an angle of $135^{\circ}$ in the centre of a $3 \mathrm{~mm}$ helium gas jet (see Fig. 1). The geometry of this arrangement allows a $100 \mu \mathrm{m} \mathrm{Al}$ foil, used for generation of transition radiation, to intercept the electron beam $15 \mathrm{~mm}$ from the exit of the gas jet. The accelerated electron beam is highly relativistic, stable and quasi-monoenergetic (see Methods and Supplementary Fig. S1). On average, the peak charge and peak energy are $15 \mathrm{pC}$ and $84 \mathrm{MeV}$ respectively. We estimate that temporal stretching during transport to the radiator due to energy spread and divergence is negligible. The beam diameter on the radiator is estimated to $90 \mu \mathrm{m}$ (FWHM) (see Methods).

The forward CTR propagates along the direction of the electron beam, through a ZnSe window (which has flat transmission in the infrared) and is collected and collimated outside the interaction chamber by a $1.5 \mathrm{~m}$-focal-length spherical silver mirror. The collimated radiation is then sent to three different diagnostics, measuring the infrared spectrum, visible spectrum and spatial distribution of the CTR.

First, we confirm that the measured radiation is indeed coherent. A lens is used to image the spatial distribution in an intermediate plane between the foil and the collecting mirror onto an absolutely calibrated 16-bit CCD (charged-coupled device) camera. An interference filter in front of the camera transmits a $\Delta \lambda=10 \mathrm{~nm}$ (FWHM) spectral band, centred at $\lambda_{0}=546 \mathrm{~nm}$. Within a $17 \mathrm{mrad}$ half-angle of collection we measure a total number of $N_{\text {ph }}=2 \times 10^{7}$ photons. No CTR signal and no electron beam is observed when the injection laser is blocked. Integration of the first term of equation (1) gives the total number of incoherent photons emitted by $N_{\mathrm{e}}$ electrons. For a bunch with charge $15 \mathrm{pC}$ and energy $84 \mathrm{MeV}$, a

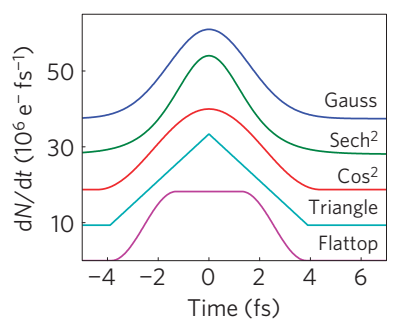

C
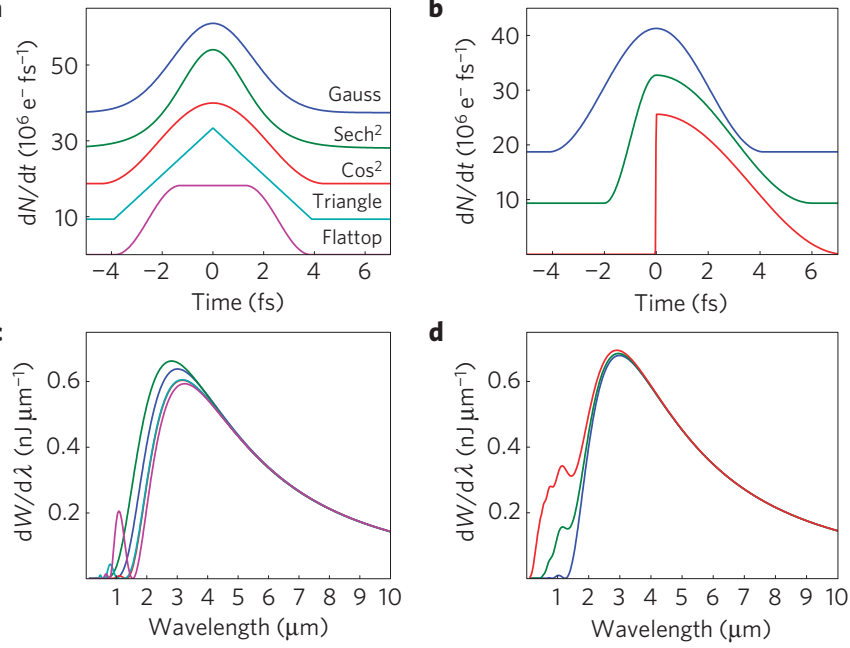

Figure 3 | Influence of the bunch shape. $\mathbf{a}, \mathbf{b}$, Symmetric (a) and asymmetric (b) temporal bunch shapes, with an r.m.s. duration of $1.5 \mathrm{fs}$. The curves are vertically offset for clarity. c,d, The corresponding CTR spectra all show a global peak around $3 \mu \mathrm{m}$. The bunch parameters used for the calculation are the measured mean values for energy, charge and divergence.

the incoherent contribution to the measured number of photons is $N_{\mathrm{ph}}$ inc $=5 \times 10^{3}$, within the solid angle of collection of the instrument. This is more than three orders of magnitude below the measured number.

The CTR spectrum is diagnosed using two instruments. An absolutely calibrated, scanning monochromator is used to measure the spectrum in a wide infrared wavelength range, $1.4-5.5 \mu \mathrm{m}$, within a half angle of collection of $2 \mathrm{mrad}$ (ref. 24). Up to 15 laser shots are averaged at each wavelength position. Simultaneously, on every laser shot, an imaging spectrometer measures the CTR spectrum in the wavelength range $0.55-1.0 \mu \mathrm{m}$, within a half angle of $17 \mathrm{mrad}$. On some shots, the spectrum is modulated. Our analysis indicates that these modulations come from the interference of CTR generated by multiple electron bunches separated by the plasma period $^{25}$ (Lundh, O. et al. manuscript in preparation). Here, we wish to determine the duration of a single bunch, and consequently shots for which spectral modulations are observed on this diagnostic are rejected from the dataset.

The measured CTR spectrum, shown in Fig. 2, has a distinct peak around $3 \mu \mathrm{m}$ which is up to seven orders of magnitude brighter than the incoherent level (grey area in the inset). The inset shows data from both the spectrometer and the monochromator, normalized to the solid angle covered by each instrument. Using the measured mean values for peak energy, bunch charge and divergence, the CTR spectrum is calculated for Gaussian bunch shapes, $f(t) \propto \exp \left[-t^{2} / 2 \sigma^{2}\right]$, with different root mean square (r.m.s.) durations $\sigma$. A good agreement, in intensity and wavelength of peak intensity, is found for a bunch duration of $\sigma=1.5 \mathrm{fs}$. For a bunch charge of $15 \mathrm{pC}$, this leads to an inferred peak current of $4 \mathrm{kA}$ (for a Gaussian pulse shape).

We now look into the influence of bunch shape on the CTR spectrum. As shown in equation (1), the bunch charge influences the intensity of the spectrum, but not the wavelength of peak intensity, $\lambda_{\mathrm{pk}}$. Likewise, one can show that for highly relativistic bunches $(v \approx c)$ and small collection angles $\left(\theta_{0} \ll \lambda / \pi \sigma_{\mathrm{r}}\right.$, where $\sigma_{\mathrm{r}}$ is the bunch radius on the radiator), the temporal bunch shape dominates and determines the form factor ${ }^{23}$. At the frequency corresponding to the peak intensity, $\omega_{\mathrm{pk}}=2 \pi c / \lambda_{\mathrm{pk}}$, we have $\mathrm{d}^{2} W / \mathrm{d} \lambda^{2}=0$, which, for a Gaussian shape, leads to a simple frequency-duration relation: $\omega_{\mathrm{pk}} \sigma=1.0$. This product has a 

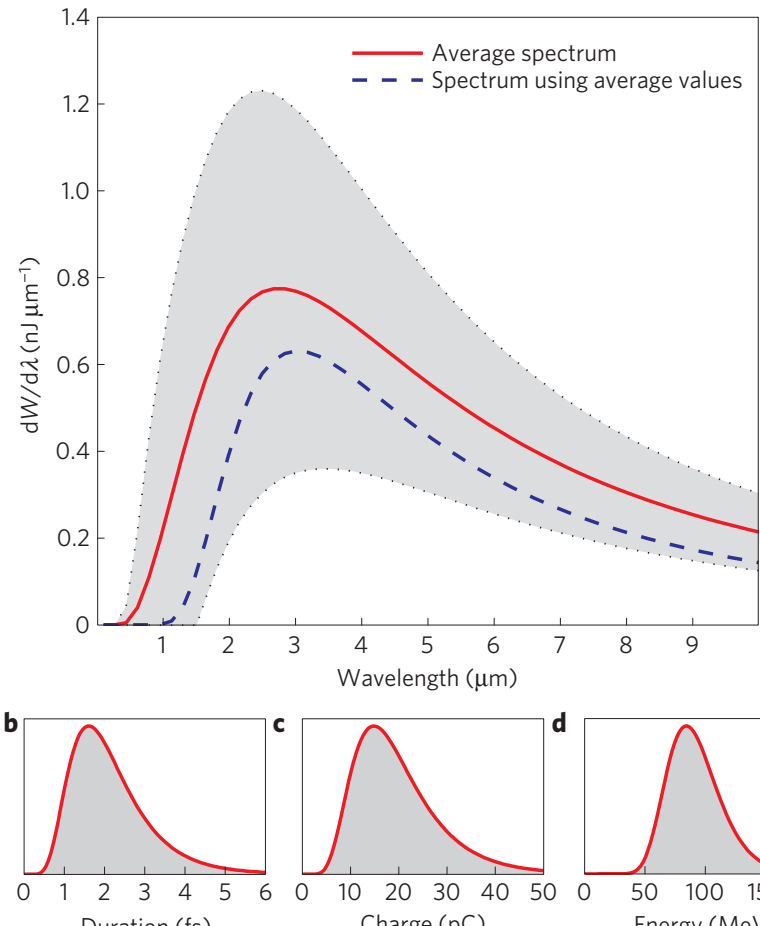

Duration (fs)
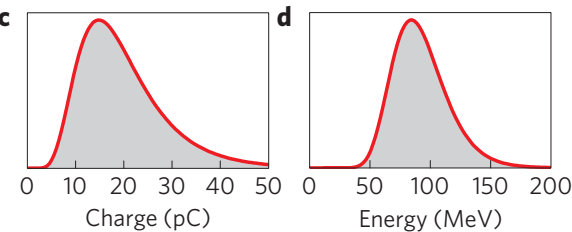

Figure 4 | Influence of statistical fluctuations. a, Average CTR spectrum (solid line) and 50\% confidence interval (dotted lines), computed from the CTR spectra of a large set of randomly generated bunches. Spectrum computed using mean values of bunch properties (dashed line). b-d, Log-normal probability density functions using hypothetical (b) and measured (c,d) mean values and variances.

relatively weak dependence on pulse shape (see Supplementary Table S1) and even for very different shapes, a global CTR peak at $3 \mu \mathrm{m}$ corresponds to r.m.s. bunch durations of 1.4-1.8 fs. Figure 3 illustrates the relatively weak influence of pulse shape on the position of the peak in the CTR spectrum.

The measurement of the CTR spectrum (Fig. 2) is done using a scanning monochromator and is averaged over a large number of shots. Therefore, we need to consider in what manner fluctuations of bunch properties (for example, charge, peak energy and duration) influence the measurement and the conclusions regarding the bunch duration. To investigate this, we choose a Monte-Carlo approach and numerically generate an ensemble of $10^{5}$ random electron bunches with Gaussian shape and using probability density functions, shown in Fig. 4b-d, to approximate the experimental variations. The CTR spectrum is then calculated for each bunch in the ensemble. In spite of large bunch-tobunch fluctuations, the average of all spectra, representing the experimental measurement, has a shape that comes relatively close to the spectrum computed using the average values of bunch properties, see Fig. 4.

We have also carefully analysed detailed effects due to accelerator dynamics (for example, beam loading) and beam transport that could influence the generation of CTR through transverse- and longitudinal phase-space correlations and bunch charge-energyduration correlations. We have found that these effects are not likely to influence the deduced value of the bunch duration by more than 10-20\% (see Supplementary Information).

Three-dimensional particle-in-cell simulations have been performed using the code CALDER (ref. 26). Parameters similar to the experiment are used, with a normalized laser vector potential $a_{0}=1.3$ for the pump pulse, $a_{1}=0.3$ for the injection pulse and an electron density $7.5 \times 10^{18} \mathrm{~cm}^{-3}$. Figure 5 a shows the phase
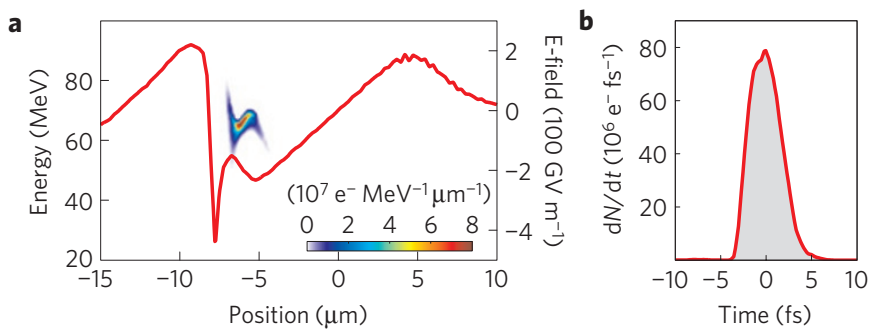

Figure 5 | Simulation results. a, Phase-space of optically injected and trapped electrons and longitudinal electric field. $\mathbf{b}$, Temporal profile of the trapped bunch.

space of electrons in the first plasma wave period, after $300 \mu \mathrm{m}$ acceleration beyond the injection point. As the electrons are highly relativistic, the length of the bunch is not expected to change dramatically during further acceleration. Figure $5 b$ shows the temporal profile of the bunch. In this simulation, the pulse shape is slightly asymmetric with rise- and fall times (between $10 \%$ and $90 \%$ of the peak current level) of 2 and 3 fs respectively. The bunch duration is $1.7 \mathrm{fs}$ (r.m.s.) or $4.4 \mathrm{fs}$ (FWHM), in good qualitative agreement with the conclusions from the measurement of the CTR spectrum. Furthermore, an independent analysis of experimental data, focusing on measured beam loading effects in an optically injected laser wakefield accelerator, indicates a bunch duration around 4 fs (ref. 27).

Finally, it should be noted that our technique has some limitations that do not allow us to exclude the existence of a longer bunch following the femtosecond bunch. Indeed, the electron energy distribution also contains a broadband component (see Supplementary Fig. S1) which might produce a longer temporal feature and emit coherent radiation at wavelengths outside the range of our detector. Our technique therefore has a limited dynamic range as longer bunches cannot be detected. In the present experiment, this component, which could be removed by an energy selective filter, contains less than $10 \mathrm{pC}$ and would therefore have a much lower peak current than the femtosecond bunch.

\section{Methods}

Optically injected laser-plasma accelerator. The experiment was carried out using the $10 \mathrm{~Hz}$ multi-terawatt Ti:sapphire laser in 'salle jaune' at Laboratoire d'Optique Appliquée (LOA), operating at a central wavelength of $820 \mathrm{~nm}$. The duration of the pulses were measured to be $30 \mathrm{fs}$ (FWHM) using spectral phase interferometry for direct electric-field reconstruction (SPIDER). The pump pulse, with an energy of $1.0 \mathrm{~J}$, was focused using an $f=1.0 \mathrm{~m}$ focal length on-axis spherical mirror. The focal spot was measured with a 12-bit CCD camera to be slightly elliptical, $17 \times 22 \mu \mathrm{m}$ at FWHM. The injection pulse, with an energy of $100 \mathrm{~mJ}$, was focused using an $f=80 \mathrm{~cm}$ focal length off-axis parabolic mirror to a $25 \mu \mathrm{m}$ (FWHM) spot. This gave an inferred intensity of $3.6 \times 10^{18} \mathrm{Wcm}^{-2}$ for the pump pulse and $1.5 \times 10^{17} \mathrm{Wcm}^{-2}$ for the injection pulse, corresponding to normalized vector potentials of $a_{0}=1.3$ and $a_{1}=0.3$ respectively. The two pulses collided at an angle of $135^{\circ}, 1 \mathrm{~mm}$ above the centre of a $3 \mathrm{~mm}$ diameter gas jet nozzle. Interferometric measurements of the gas jet density profile at this height revealed a $2.1 \mathrm{~mm}$ plateau surrounded by $700 \mu \mathrm{m}$ gradients on each side. Helium gas was used at a backing pressure of 6 bar and provided a fully ionized plasma electron density of $n_{\mathrm{e}}=1 \times 10^{19} \mathrm{~cm}^{-3}$.

CTR generation and characterization. Forward CTR was generated at the rear surface of a $100 \mu \mathrm{m} \mathrm{Al}$ foil which also served to stop the pump laser beam and prevent parasitic radiation from reaching the detection system. Before every shot, the foil was translated to provide unexposed material for the electron beam. The electron beam size on the foil was estimated to $90 \pm 24 \mu \mathrm{m}$ from the measured beam divergence. The $\mathrm{Al}$ foil was positioned $0.5 \mathrm{~m}$ from the $\mathrm{ZnSe}$ chamber window and $1.5 \mathrm{~m}$ from the collecting Ag mirror. For the photon counting measurement, an $f=200 \mathrm{~mm}$ focal length, $f / 4$ achromatic doublet lens was used to illuminate $\sim 2.6 \times 10^{4}$ pixels on an absolutely calibrated 16-bit CCD camera (Andor DV420). For the spectral measurements, the CTR beam was split using a ZnSe window with a small wedge. The front Fresnel reflection was focused through the slit of a Czerny-Turner imaging spectrometer (Oriel MS127i) using an $f=200 \mathrm{~mm}$ focal length, $f / 4$ achromatic doublet lens. A grating with 400 lines/mm was 
used for dispersion and a 16-bit CCD camera (Andor DV420) was used for detection. The instrumental spectral response function was determined using a calibrated Xe lamp, a HeNe laser and a power meter. The transmission through the $\mathrm{ZnSe}$ exit window was measured and corrected for. The parallel CTR beam transmitted through the ZnSe beam splitter was focused using an $f=100 \mathrm{~mm}$ off-axis Au parabolic mirror through the fully opened slit of the specially built monochromator. A ZnSe prism with an apex angle $40^{\circ}$ was used at $51^{\circ}$ angle of incidence for dispersion. An $f=270 \mathrm{~mm}$ off-axis parabolic Au mirror was used for both collimation and refocusing and a retro-reflecting scanning Au mirror was used for wavelength selection. The InSb photovoltaic detector (Hamamatsu P5968) was absolutely calibrated using a laser diode and a power meter. The dispersion function was determined by using a blackbody source and a set of interference filters. The radiation propagated a total of $2.5 \mathrm{~m}$ in air before reaching the InSb detector. The absorption of $2.5 \mathrm{~m}$ of air was evaluated using HITRAN absorption data. It was below $15 \%$ for all measured wavelengths. The spectral resolution was determined by the size of the detector and was $\Delta \lambda / \lambda \leq 0.1$ within the range $1.4-5.5 \mu \mathrm{m}$.

Electron beam characterization. The electron beam was dispersed by a round electromagnetic dipole, providing an effective magnetic field of $1.6 \mathrm{~T}$ over $25 \mathrm{~mm}$. A doublet lens imaged the scintillation from a LANEX phosphor screen onto a 16-bit CCD camera (Andor DV434). The arrangement was absolutely calibrated and is described in detail in ref. 28. With the magnetic field turned off and the radiato foil removed from the beam path we measured a divergence of $6.0 \pm 1.6 \mathrm{mrad}$ (FWHM) and a pointing fluctuation of $2.2 \mathrm{mrad}$ (r.m.s.). With the radiator in the path, we measured a divergence of $15 \pm 7 \mathrm{mrad}$ (FWHM). The spectral resolution was limited by the electron beam divergence. At $84 \mathrm{MeV}$ it was $12 \%$ with the radiator in the path. For the shots included in the CTR study, we measured: peak energy $84 \pm 21 \mathrm{MeV}$, energy spread $21 \pm 17 \mathrm{MeV}$ (FWHM), peak charge $15 \pm 7 \mathrm{pC}$, total charge $23 \pm 9 \mathrm{pC}$.

Received 12 March 2010; accepted 2 November 2010; published online 9 January 2011

\section{References}

1. Tajima, T. \& Dawson, J. M. Laser electron accelerator. Phys. Rev. Lett. 43, 267-270 (1979).

2. Faure, J. et al. A laser-plasma accelerator producing monoenergetic electron beams. Nature 431, 541-544 (2004).

3. Geddes, C. G. R. et al. High-quality electron beams from a laser wakefield accelerator using plasma-channel guiding. Nature 431, 538-541 (2004).

4. Mangles, S. P. D. et al. Monoenergetic beams of relativistic electrons from intense laser-plasma interactions. Nature 431, 535-538 (2004).

5. Rechatin, C. et al. Controlling the phase-space volume of injected electrons in a laser-plasma accelerator. Phys. Rev. Lett. 102, 164801 (2009).

6. Fritzler, S. et al. Emittance measurements of a laser-wakefield-accelerated electron beam. Phys. Rev. Lett. 92, 165006 (2004).

7. Leemans, W. P. et al. GeV Electron beams from a centimetre-scale accelerator. Nature Phys. 2, 696-699 (2006).

8. Hafz, N. et al. Stable generation of GeV-class electron beams from self-guided laser-plasma channels. Nature Photon. 2, 571-577 (2008).

9. Faure, J. et al. Controlled injection and acceleration of electrons in plasma wakefields by colliding laser pulses. Nature 444, 737-739 (2006)

10. Schlenvoigt, H-P. et al. A compact synchrotron radiation source driven by a laser-plasma wakefield accelerator. Nature Phys. 4, 130-133 (2008).

11. Fuchs, M. et al. Laser-driven soft-x-ray undulator source. Nature Phys. 5, 826-829 (2009).

12. Grüner, F. et al. Design considerations for table-top, laser-based vuv and X-ray free electron lasers. Appl. Phys. B 86, 431-435 (2007).
13. Nakajima, K. Compact x-ray sources: Towards a table-top free-electron laser. Nature Phys. 4, 92-93 (2008).

14. Malka, V. et al. Principles and applications of compact laser-plasma accelerators. Nature Phys. 4, 447-453 (2008).

15. Mangles, S. P. D. et al. Laser-wakefield acceleration of monoenergetic electron beams in the first plasma-wave period. Phys. Rev. Lett. 96, 215001 (2006).

16. Liu, Y. et al. Experimental observation of femtosecond electron beam microbunching by inverse free-electron-laser acceleration. Phys. Rev. Lett. 80, 4418-4421 (1998)

17. Tremaine, A. et al. Observation of self-amplified spontaneous-emissioninduced electron-beam microbunching using coherent transition radiation. Phys. Rev. Lett. 81, 5816-5819 (1998).

18. Ding, Y. et al. Measurements and simulations of ultralow emittance and ultrashort electron beams in the linac coherent light source. Phys. Rev. Lett. 102, 254801 (2009).

19. Leemans, W. P. et al. Observation of terahertz emission from a laser-plasma accelerated electron bunch crossing a plasma-vacuum boundary. Phys. Rev. Lett. 91, 074802 (2003).

20. van Tilborg, J. et al. Temporal characterization of femtosecond laser-plasma-accelerated electron bunches using terahertz radiation. Phys. Rev. Lett. 96, 014801 (2006).

21. Debus, A. D. et al. Electron bunch length measurements from laser- accelerated electrons using single-shot THz time-domain interferometry. Phys. Rev. Lett. 104, 084802 (2010).

22. Shibata, Y. et al. Observation of coherent transition radiation at millimeter and submillimeter wavelengths. Phys. Rev. A 45, R8340-R8343 (1992).

23. Faure, J., Glinec, Y., Gallot, G. \& Malka, V. Ultrashort laser pulses and ultrashort electron bunches generated in relativistic laser-plasma interaction. Phys. Plasmas 13, 056706 (2006).

24. Lim, J. et al. Femto-second ultrashort laser wakefield electron bunch-duration measurements: A prism-based dispersion visible-to-IR spectrometer. Proc. SPIE 7359, 735919 (2009).

25. Glinec, Y., Faure, J., Norlin, A., Pukhov, A. \& Malka, V. Observation of fine structures in laser-driven electron beams using coherent transition radiation. Phys. Rev. Lett. 98, 194801 (2007).

26. Lefebvre, E. et al. Electron and photon production from relativistic laser-plasma interactions. Nucl. Fusion 43, 629-633 (2003).

27. Rechatin, C. et al. Characterization of the beam loading effects in a laser plasma accelerator. New J. Phys. 12, 045023 (2010).

28. Glinec, Y. et al. Absolute calibration for a broad range single shot electron spectrometer. Rev. Sci. Instrum. 77, 103301 (2006).

\section{Acknowledgements}

We acknowledge the support of the European Research Council for funding the PARIS ERC project (contract 226424) and the support from EC FP7 LASERLAB-EUROPE/LAPTECH (contract 228334).

\section{Author contributions}

O.L., J.L., C.R., L.A., A.B-I., J-P.G. and J.F. carried out the experiment. O.L. and C.R. analysed the data. X.D. and E.L. carried out the simulations. J.L., O.L., G.G. and J.F. developed the infrared spectrometer. V.M. and J.F. guided the project.

\section{Additional information}

The authors declare no competing financial interests. Supplementary information accompanies this paper on www.nature.com/naturephysics. Reprints and permissions information is available online at http://npg.nature.com/reprintsandpermissions. Correspondence and requests for materials should be addressed to O.L. or V.M. 